\title{
Polarization of $21 \mathrm{~cm}$ Radiation from the Epoch of Reionization
}

\section{Citation}

Babich, Daniel, and Abraham Loeb. 2005. "Polarization of $21 \mathrm{Cm}$ Radiation from the Epoch of Reionization." The Astrophysical Journal 635 (1): 1-10. https://doi.org/10.1086/497297.

\section{Permanent link}

http://nrs.harvard.edu/urn-3:HUL.InstRepos:41393441

\section{Terms of Use}

This article was downloaded from Harvard University's DASH repository, and is made available under the terms and conditions applicable to Other Posted Material, as set forth at http:// nrs.harvard.edu/urn-3:HUL.InstRepos:dash.current.terms-of-use\#LAA

\section{Share Your Story}

The Harvard community has made this article openly available.

Please share how this access benefits you. Submit a story. 


\title{
POLARIZATION OF $21 \mathrm{~cm}$ RADIATION FROM THE EPOCH OF REIONIZATION
}

\author{
Daniel Babich ${ }^{1,2}$ AND ABraham Loeb ${ }^{2,3}$ \\ Received 2005 May 17; accepted 2005 August 15
}

\begin{abstract}
We consider the polarization of $21 \mathrm{~cm}$ line radiation from the epoch of reionization due to both intrinsically polarized emission and secondary mechanisms. We argue that Thomson scattering of the $21 \mathrm{~cm}$ quadrupole by the reionized universe is likely to produce the largest effect. The $21 \mathrm{~cm}$ quadrupole is sourced by baryonic density fluctuations and the fluctuations in the ionization fraction due to discrete $\mathrm{H}$ in regions. Since Thomson scattering produces only $E$-type polarization and is achromatic, its unique fingerprint could in principle be separated from foregrounds associated with polarized synchrotron emission, which should not be correlated with the cosmic signal. We estimate that Poisson fluctuations of $\mathrm{H}$ II regions at the end of reionization $\left(z_{R} \sim 6-20\right)$ produce a brightness temperature-polarization cross-correlation signal of $\sim 0.1-0.3 \mathrm{mK}$ on angular scales of tens of arcminutes. This cross-correlation signal is within the instrument sensitivities of the future Square Kilometre Array (SKA) and close to the sensitivities of the forthcoming Mileura Widefield Array (MWA) and Low Frequency Array (LOFAR).
\end{abstract}

Subject headings: cosmology: theory — diffuse radiation — intergalactic medium — polarization radio lines: general

Online material: color figures

\section{INTRODUCTION}

Measurements of the power spectrum of $21 \mathrm{~cm}$ brightness fluctuations could constrain the complex processes that reionized the universe (Sunyaev \& Zel'dovich 1975; Hogan \& Rees 1979; Scott \& Rees 1990; Tozzi et al. 2000; Madau et al. 1997; Furlanetto et al. 2004; Barkana \& Loeb 2005b), as well as the basic cosmological parameters (Loeb \& Zaldarriaga 2004; Barkana \& Loeb 2005a, 2005c). By tuning low-frequency radio arrays to different frequencies, one could observe the intergalactic medium (IGM) at different redshift slices as it evolved through the epoch of reionization. Observations of the temperature brightness power spectrum will allow us to infer the baryonic power spectrum and the peculiar velocity field at high redshifts, as well as the mean cosmic ionization fraction and fluctuations in the ionization fraction due to discrete $\mathrm{H}$ II regions. Constraints on these quantities will test models of reionization, in particular the spectra and evolution of the first sources of ionizing radiation (Barkana \& Loeb 2001). Several low-frequency arrays are currently under construction for the purpose of mapping neutral hydrogen during the epoch of reionization; ${ }^{4}$ these include the Primeval Structure Telescope (PAST), ${ }^{5}$ the Mileura Widefield Array (MWA), ${ }^{6}$ and the Low Frequency Array (LOFAR), ${ }^{7}$ which would establish the grounds for the construction of the Square Kilometre Array (SKA). ${ }^{8}$

Almost all theoretical work thus far has focused on the brightness temperature fluctuations of the redshifted $21 \mathrm{~cm}$ signal. The exact shape of the brightness temperature fluctuation

\footnotetext{
1 Department of Physics, Harvard University, 17 Oxford Street, Cambridge, MA 02138; babich@physics.harvard.edu.

2 Harvard-Smithsonian Center for Astrophysics, 60 Garden Street, Cambridge, MA 02138; aloeb@cfa.harvard.edu.

3 Department of Astronomy, Harvard University, 60 Garden Street, Cambridge, MA 02138.

4 See http://space.mit.edu/eor-workshop/.

5 See http://web.phys.cmu.edu/ past.

6 See http://web.haystack.mit.edu/arrays/MWA.

7 See http://www.lofar.org.

8 See http://www.skatelescope.org.
}

power spectrum during reionization depends on the size and spatial distribution of the $\mathrm{H}$ II regions and should peak on the scale corresponding to the characteristic $\mathrm{H}$ II region size. On scales below this characteristic size the power in the fluctuations should decline. It is difficult to model this power spectrum without introducing considerable astrophysical uncertainties, involving the formation and spectra of the first stars and quasars (Wyithe \& Loeb 2003), feedback effects that could impede the formation of future sources (Furlanetto \& Loeb 2005), and shadowing by compact low-mass halos (Barkana \& Loeb 2002; Shapiro et al. 2004). Ultimately, hydrodynamic simulations with radiative transfer will be the most reliable method for following the evolution of the $\mathrm{H}$ II regions during the epoch of reionization. However, even these will inevitably be sensitive to uncertain details about the processes of star formation and quasar accretion, which supply the ionizing photons. At present, the large dynamical range needed in order to reliably follow radiative transfer in simulated boxes with periodic boundary conditions (Barkana \& Loeb 2004) is still orders of magnitude beyond the values that state-of-the-art codes (Gnedin 2004; Sokasian et al. 2004) are able to achieve.

In this paper we examine the polarization of the redshifted $21 \mathrm{~cm}$ radiation. The polarization could result from (1) intrinsic properties of the sources and (2) processes that polarize the radiation as it travels toward the observer (secondary mechanisms). In the first category we analyze processes that produce a nonisotropic population of the hyperfine triplet state, which upon spontaneous decay could produce $21 \mathrm{~cm}$ polarization. Cooray \& Furlanetto (2005) already considered the intrinsic polarization due to the Zeeman effect. They concluded that the effect was most likely too small to be observed for realistic values of the intergalactic magnetic fields at high redshifts. The second category of polarization mechanisms is identical to the secondary processes that produce polarization in the cosmic microwave background (CMB; Zaldarriaga 1997; Hu 2000). Here we focus on the generation of polarization out of the free-streaming $21 \mathrm{~cm}$ anisotropies through Thomson scattering by the reionized universe. 
The polarization anisotropies carry important new information about reionization. For example, if the dominant source of polarization is Thomson scattering in the reionized universe, then the $21 \mathrm{~cm}$ polarization would gauge the topology of ionized bubbles near the end of reionization, as well as the optical depth to electron scattering after the bubbles overlapped. The latter measurement would help remove degeneracies among cosmological parameters from the $\mathrm{CMB}$ data alone and in particular substantiate the inference about the optical depth from the Wilkinson Microwave Anisotropy Probe (WMAP; Spergel et al. 2003). The associated constraints on the growth and morphology of $\mathrm{H}$ II regions during the epoch of reionization (Zahn et al. 2005; McQuinn et al. 2005) could remove the existing tension between the conclusions drawn from the CMB data (Spergel et al. 2003) and the sizes of quasar H II regions (Wyithe \& Loeb 2004a; Mesinger \& Haiman 2004).

An additional reason to study the polarization of the $21 \mathrm{~cm}$ signal is that the expected low-frequency foreground is several orders of magnitude brighter than the redshifted $21 \mathrm{~cm}$ signal (Di Matteo et al. 2002; Oh \& Mack 2003). The foregrounds are expected to be smooth in frequency space, while the signal due to $21 \mathrm{~cm}$ emission should fluctuate between narrowly spaced bands, so it was argued that the small signal could be extracted by taking the differences between maps at narrowly separated frequency bands (Zaldarriaga et al. 2004; Morales \& Hewitt 2004). Since the foregrounds are expected to be polarized, a definite prediction for the polarization, both the signal and foreground, would further help us disentangle the signal from the foregrounds. If the polarization signal is observable, it could be of a particular parity type (e.g., $E$-type or $B$-type; see Zaldarriaga $\&$ Seljak [1997] for a review of polarization). This additional information could also be used to separate the signal from the foregrounds. Even if the polarization signal is not expected to be observable by a particular experiment, we can still use the prediction that any polarization detected by such an experiment should not correlate with the inferred signal in order to reduce the foreground contamination.

The organization of the paper is as follows. In $\S 2$ we review the possible mechanisms that could produce a polarized signal. In $\S 3$ we present numerical results for secondary polarization anisotropies due to Thomson scattering in the reionized universe, which we expect to be the dominant source of polarization. Finally, $\S 4$ summarizes our main conclusions. We adopt the standard $\Lambda$ CDM cosmological model consistent with the WMAP (Spergel et al. 2003) data $\left(\Omega_{b}=0.044, \Omega_{m}=0.27, \Omega_{v}=0.73\right.$, $n=1, \sigma_{8}=0.9$, and $h=0.72$ ), and we show results for different values of the reionization optical depth and the redshift of reionization. For simplicity, we ignore helium and assume a pure hydrogen plasma. We also focus on $21 \mathrm{~cm}$ emission, since the spin temperature of hydrogen is likely to exceed the CMB temperature of $T_{\mathrm{CMB}}=2.7(1+z) \mathrm{K}$ due to heating by X-rays; the heating is expected to occur soon after the first sources light up and long before the universe gets reionized, since it requires only $\lesssim 10^{-2} \mathrm{eV}$ (instead of $\gtrsim 10 \mathrm{eV}$ for ionization) per baryon.

\section{POLARIZATION MECHANISMS}

As already mentioned, the mechanisms that are capable of producing polarization in the $21 \mathrm{~cm}$ line divide into two broad classes: (1) intrinsic emission and (2) secondary mechanisms. In order to produce polarized intrinsic emission, the triplet state of the hyperfine splitting needs to be excited in a nonisotropic way (e.g., through pumping by an anisotropic radiation field or splitting by a magnetic field, which introduces a preferred direction), and then the spontaneous decay of this excited state will produce polarized light. It is also necessary that collisional processes, which drive the system to thermal equilibrium, are not more rapid than the spontaneous decay and anisotropic triplet pumping rates (Zygelman 2005).

Even if the intrinsic emission is unpolarized, secondary mechanisms such as Thomson or resonant scattering along that line of sight would produce polarization in an analogous manner to the secondary production of polarization in the CMB. In this case, the polarization is sourced by an incident quadrupole brightness moment in the scatterer's frame of reference. The optical depth to electron scattering inferred by WMAP, $\tau \sim$ $0.17 \pm 0.04$ (Spergel et al. 2003), implies that secondary scattering could produce a nonnegligible effect. The fundamental difference between the scattered $21 \mathrm{~cm}$ polarization and reionization bump for the CMB polarization is the source of the incident quadrupole. The incident quadrupole in the CMB case originates at the surface of last scattering at the redshift of recombination $\left(z=10^{3}\right)$ and is due to the projection and freestreaming of the correlated CMB temperature fluctuations. The free-streaming distance is always the horizon size as seen by the scatterer. While the basic physical process is identical, these details are changed in the $21 \mathrm{~cm}$ case. First, the free-streaming distance from the emitter to the scatterer is not always the horizon size but is set by the frequency of the observed line radiation. The dominant sources of the incident quadrupole during reionization are likely to be baryonic density fluctuations in the $\mathrm{H}$ I gas and ionization fraction fluctuations based on the topology of $\mathrm{H}$ II regions. The optical depth for resonant $21 \mathrm{~cm}$ absorption is typically smaller than 1\% (Carilli et al. 2002; Furlanetto \& Loeb 2002) and is ignored in our discussion.

\subsection{Intrinsic Emission}

Isotropic symmetry needs to be broken in order produce net polarization (i.e., a preferred direction) in $21 \mathrm{~cm}$ line emission from an ensemble of hydrogen atoms. There are two simple ways to break isotropy: (1) Zeeman splitting of the triplet state due to a directional magnetic field and (2) the WouthuysenField effect due to an anisotropic Ly $\alpha$ radiation field. The possibility of the Zeeman effect has already been considered by Cooray \& Furlanetto (2005). In order for the signal to be measurable by SKA, a coherent field strength of $\gtrsim 100 \mu \mathrm{G}$ on megaparsec scales is required at $z \sim 6-10$, with an energy density comparable to that of the CMB at that time. Such a field strength is well above current Faraday rotation limits (Vallée 2004) on the intergalactic field outside galaxy clusters [keeping in mind the adiabatic enhancement factor of $(1+z)^{2}$ to high redshifts] and is orders of magnitude above theoretical expectations from astrophysical sources (Furlanetto \& Loeb 2001; Kulsrud et al. 1997).

The Wouthuysen-Field effect is the mechanism that couples the gas temperature to the hyperfine spin temperature through the absorption and emission of Ly $\alpha$ photons (Wouthuysen 1952; Field 1959). There is a finite probability for a $2 p$ state, which was reached by Ly $\alpha$ absorption by the singlet $1 s$ state, to spontaneously decay into the triplet $1 s$ state. This process will convert a fraction of the singlet states to triplet states. When the Ly $\alpha$ scattering rate is high, the spin temperature, which determines the relative populations of the singlet and triplet states, will equal the kinetic temperature of the gas (which sets the "color temperature" of the Ly $\alpha$ photons). During reionization the kinetic temperature of the gas is heated (e.g., by a 
small amount of $\mathrm{X}$-rays produced by the first compact objects) to a value much greater than the CMB temperature. The corresponding increase in the spin temperature allows the $21 \mathrm{~cm}$ line to be seen in emission during the late stage of reionization.

If the $\operatorname{Ly} \alpha$ radiation as seen by a hydrogen atom has a quadrupole moment, then the $21 \mathrm{~cm}$ line emitted by this atom will be polarized (as long as atomic collisions can be neglected; see Zygelman 2005). The basic idea is that continuum radiation from the first high-redshift sources will be redshifted into the blue wing of the $\mathrm{Ly} \alpha$ line profile. This radiation field could be anisotropic because the ionizing sources are highly clustered at high redshifts (Wyithe \& Loeb 2005). Unfortunately, the large optical depth near the Ly $\alpha$ resonance $\left(\sim 10^{5}\right.$ for a neutral IGM) quickly isotropizes the incident radiation as it is redshifted through the line profile by the cosmological expansion. Therefore, only the photons in the far blue wing of the Ly $\alpha$ line profile are expected to produce a nonisotropic pumping of the triplet state distribution. However, these photons are much less likely to scatter than the isotropized photons near the center of the line. Even in the vicinity of a bright quasar, the anisotropy of the incident radiation would induce polarization only for the neutral IGM within a thin skin outside the quasar's H II region whose spatial width is of the order of the mean free path at the center of the thermally broadened Ly $\alpha$ line, $\lesssim 0.1 \mathrm{pc}$ (Wyithe \& Loeb 2004c). We conclude that pumping by an anisotropic Ly $\alpha$ radiation field is unlikely to produce observable intrinsically polarized $21 \mathrm{~cm}$ line radiation.

\subsection{Secondary Mechanisms}

Thomson scattering by free electrons along the line of sight can polarize intrinsically unpolarized emission if the radiation field as seen by the scattering electrons possesses a quadrupole moment. The inhomogeneous distribution of the emitting $\mathrm{H}$ I gas naturally generates a $21 \mathrm{~cm}$ quadrupole as the radiation freestreams toward the free electrons in the reionized universe. The $21 \mathrm{~cm}$ emission originates from the neutral fraction of the IGM, so fluctuations in the ionization fraction will also produce fluctuations in the $21 \mathrm{~cm}$ brightness. There should be fluctuations in the redshift of reionization along different directions due to large-scale inhomogeneities (Barkana \& Loeb 2004; Wyithe \& Loeb 2004c). Unfortunately, the detailed correlation function and size distribution of $\mathrm{H}$ II regions depend strongly on uncertain details of the astrophysics of star and black hole formation. This dependence introduces inherent uncertainties into the theoretical modeling of the ionization fraction power spectrum (Furlanetto et al. 2004; McQuinn et al. 2005).

The end of reionization, however, offers a special regime in which robust model-independent predictions can be made. This was demonstrated by Wyithe \& Loeb (2004b), who derived the characteristic size of individual $\mathrm{H}$ II regions just before their final overlap based on the extended Press-Schetcher theory. This derivation, which was based on the general physical considerations of cosmic variance and light propagation delay, eliminates much of the dependence on the astrophysical details. We therefore incorporate this more robust approach and derive the $21 \mathrm{~cm}$ polarization power spectrum from it. For simplicity, we assume that Thomson scattering starts after the final overlap of the $\mathrm{H}$ II regions, since most of the Thomson scattering optical depth is naturally in the regime in which the filling fraction of ionized regions is substantial. In our model, the $21 \mathrm{~cm}$ quadrupole is sourced by the Poisson fluctuations of the $\mathrm{H}$ II regions at the end of reionization. This effect will produce the largest signal; however, for completeness in $\S 3$ we also display results from the baryon density fluctuation power spectrum.
The two Stokes parameters $Q$ and $U$ of polarized radiation can be decomposed into the rotationally invariant $E$-type and $B$-type fields (Seljak \& Zaldarriaga 1996). Due to parity invariance, perturbations, which rotationally transform as scalars and therefore lack any handedness, will only produce $E$-type polarization. The $21 \mathrm{~cm}$ quadrupole is sourced by baryon density and ionization fraction fluctuations and should only produce $E$-type polarization, since the induced polarization is azimuthally symmetric about the initial perturbation wavevector. This is an important conclusion because it can be used to eliminate polarized foreground emission and instrumental systematics.

Propagation effects, such as gravitational lensing (Zaldarriaga \& Seljak 1998) and Faraday rotation (Kosowsky \& Loeb 1996; Kosowsky et al. 2005), will convert the $E$-type polarization produced by Thomson scattering into $B$-type polarization. The amplitude of $B$-modes produced by gravitational lensing should not be significant (Zaldarriaga \& Seljak 1998); however, the effect of Faraday rotation could be quite substantial at the relevant low frequencies. It is difficult to estimate the properties of the appropriate rotation measures, since they depend on the magnetic field amplitude and coherence structure, not just in the Galactic interstellar medium, but also in the high-redshift IGM. Nevertheless, the effect of Faraday rotation has a known frequency dependence, and it is possible that the developed techniques for foreground removal could be adapted to address this challenge.

While calculating the effect of Thomson scattering on the incident $21 \mathrm{~cm}$ quadrupole, we ignore the inhomogeneities present in the distribution of scattering electrons. For simplicity, we calculate the expected polarization signal assuming a sudden uniform reionization. In reality, prior to the end of reionization, the ionization fraction is less than unity, with full ionization only in localized $\mathrm{H}$ II regions around biased clusters of sources. Since the ionized bubbles contain free electrons, they would also scatter the incident quadrupole and produce polarization. The resulting visibility function will be inhomogeneous, and scattering will be able to produce $B$-mode polarization. In all calculations of the effect for the $\mathrm{CMB}$, the produced $B$-mode power spectrum was confined to small scales and was several orders of magnitude smaller in amplitude than the large-scale $E$-mode polarization (Hu 2000); we do not expect this conclusion to differ for the $21 \mathrm{~cm}$ line radiation, so our working assumption is that the large-scale polarization only depends on the optical depth of the homogeneous universe.

In $\S 3$ we demonstrate that the effect of assuming an instantaneous, instead of gradual, homogeneous reionization history is modest by showing that the polarization power spectrum can be well approximated by a polarization power spectrum calculated from an instantaneous reionization history. Of course, the two power spectra are not identical; however, the appropriate power spectrum error bars, both cosmic variance and instrumental noise, must be included in making the comparison. Similarly, it has been noted that the reionization history can be approximated as instantaneous for low-sensitivity CMB polarization measurements (such as WMAP), but for upcoming nearly cosmic variance limited measurements (such as Planck) a better model of reionization is necessary (Haiman $\&$ Holder 2003; Holder et al. 2003). Since the introduced theoretical uncertainties should be modest compared to the large instrumental noise and foreground contamination, we use the assumption of instantaneous reionization in this paper.

As is customary, we write down the polarization transfer function in the line-of-sight formalism (Seljak \& Zaldarriaga 1996; Hu \& White 1997), which is the Legendre expansion 
of the $E$-type polarization induced by a single Fourier mode perturbation,

$$
\Delta_{l}^{E}(k, \nu)=\frac{3}{4} \sqrt{\frac{(l+2) !}{(l-2) !}} \int_{0}^{\eta_{R}} d \eta \frac{g(\eta)}{\eta^{2} k^{2}} j_{l}(k \eta) \Pi(k, \eta, \nu),
$$

where $k$ is the primordial wavevector, $l$ is the observed Legendre mode, and $\eta$ is conformal look-back time, defined as

$$
\eta(z)=\int_{0}^{z} \frac{d z^{\prime}}{H\left(z^{\prime}\right)} .
$$

Here we also define the visibility function, which is the probability that a photon last scattered at $\eta$, as

$$
g(\eta)=\frac{d \tau}{d \eta} e^{-\tau(\eta)}
$$

where

$$
\frac{d \tau}{d \eta}=\sigma_{\mathrm{T}} x_{e}(z) n_{b}(1+z)^{2}
$$

$\sigma_{\mathrm{T}}$ is the cross section for Thomson scattering, $x_{e}(z)$ is the ionization fraction, and $n_{b}$ is the average baryon number density today. The incident quadrupole on the scatterer is $\Pi(k, \eta)$, and $\nu$ is the observed frequency of the $21 \mathrm{~cm}$ line. Since Thomson scattering is achromatic, the change in the observed frequency will simply be due to the cosmological redshift; thus, we can relate the observed frequency to the rest-frame frequency, $\nu_{0}=$ $1.4 \mathrm{GHz}$, as $\nu=\nu_{0} /\left(1+z_{E}\right)$, where $z_{E}$ is the emission redshift.

The smooth intergalactic $\mathrm{H}$ I gas has a $21 \mathrm{~cm}$ optical depth (Field 1959; Bharadwaj \& Ali 2004; Barkana \& Loeb 2005a)

$$
\tau=\frac{3 c^{3} \hbar A_{10} x_{\mathrm{H}} n_{b}(1+\delta)(1+z)^{3}}{16 k_{\mathrm{B}} \nu_{0}^{2} T_{S} H(z)}\left[1-\frac{(1+z)}{H(z)} \frac{d v_{r}}{d r}\right]
$$

where $A_{10}$ is the spontaneous emission coefficient of the hyperfine transition, $T_{S}$ is the spin temperature that determines the relative populations of the triplet and singlet states, $x_{\mathrm{H}}$ is the neutral fraction of the hydrogen gas, and $\delta$ is the baryonic overdensity. Here $d v_{r} / d r$ is the radial gradient of the line-of-sight peculiar velocity of the $\mathrm{H}$ I gas. The optical depth is proportional to the path length over which a $21 \mathrm{~cm}$ photon resonates with hydrogen atoms, which is in turn inversely proportional to the rate by which the Doppler shift of the medium changes along its path. Equation (5) contains contributions to the optical depth from both the cosmological expansion and the peculiar velocity of the gas. The fluctuation in the brightness temperature, calculated from the equation of radiative transfer, is

$$
\begin{aligned}
\delta T_{b}(z) & =\frac{T_{S}-T_{\mathrm{CMB}}}{(1+z)} \tau \\
& \approx 23\left[1+\left(1+\mu^{2}\right) \delta\right] x_{\mathrm{H}} \frac{\left(T_{S}-T_{\mathrm{CMB}}\right)}{T_{S}}\left(\frac{1+z}{10}\right)^{1 / 2} \mathrm{mK}
\end{aligned}
$$

where $\mu$ is defined as $\mu=\hat{\boldsymbol{k}} \cdot \hat{\boldsymbol{n}}$, the cosine of the angle between the wavevector of the primordial perturbation and the line of sight. Since the secondary polarization mechanism is achro- matic, we simply consider the dimensionless brightness temperature fluctuation, $\psi$, which is defined as

$$
\psi=\left[1+\left(1+\mu^{2}\right) \delta\right] x_{\mathrm{H}} \frac{\left(T_{S}-T_{\mathrm{CMB}}\right)}{T_{S}} .
$$

In the limit $T_{S} \gg T_{\mathrm{CMB}}$, which we expect to hold late in reionization, this can simply be expressed as $\psi=x_{\mathrm{H}}\left[1+\left(1+\mu^{2}\right) \delta\right]$.

These correlated brightness temperature fluctuations will produce a quadrupole incident on the scatterers because freestreaming of fluctuations transfers power from low Legendre modes to higher ones ( $\mathrm{Hu} \&$ White 1997). Performing this projection and free-streaming, the incident dimensionless quadrupole can be expressed as (Zaldarriaga 1997; Hu 2000)

$$
\begin{aligned}
\Pi(k, \eta, \nu)= & \int_{-1}^{+1} d \mu P_{2}(\mu) e^{i \mu k\left(\eta_{E}-\eta\right)} \psi\left(k, \eta_{E}\right), \\
= & j_{2}\left[k\left(\eta_{E}-\eta\right)\right] x_{\mathrm{H}}\left(k, \eta_{E}\right)\left[1+\delta\left(k, \eta_{E}\right)\right] \\
& -j_{2}^{\prime \prime}\left[k\left(\eta_{E}-\eta\right)\right] x_{\mathrm{H}}\left(\eta_{E}\right) \delta\left(k, \eta_{E}\right),
\end{aligned}
$$

where $\eta_{E}$ is simply the conformal distance to the redshift of emission, $z_{E}$. Each prime denotes a derivative of the spherical Bessel function; the derivative term in equation (10) is produced by the peculiar velocity term in equation (8).

Unlike the calculation of CMB anisotropies, we need to define two separate transfer functions for the polarization produced by the baryonic density fluctuations,

$$
\begin{aligned}
& { }_{\delta} \Delta_{l}^{E}(k, \nu)=\frac{3}{4} \sqrt{\frac{(l+2) !}{(l-2) !}} \\
& \quad \times \int_{0}^{\eta_{R}} d \eta \frac{g(\eta)}{\eta^{2} k^{2}} j_{l}(k \eta)\left\{j_{2}\left[k\left(\eta_{E}-\eta\right)\right]-j_{2}^{\prime \prime}\left[k\left(\eta_{E}-\eta\right)\right]\right\},
\end{aligned}
$$

and the ionization fraction fluctuations,

$$
{ }_{x} \Delta_{l}^{E}(k, \nu)=\frac{3}{4} \sqrt{\frac{(l+2) !}{(l-2) !}} \int_{0}^{\eta_{R}} d \eta \frac{g(\eta)}{\eta^{2} k^{2}} j_{l}(k \eta) j_{2}\left[k\left(\eta_{E}-\eta\right)\right] .
$$

We must make this distinction, since we cannot simply model the ionization fraction fluctuations in terms of the baryonic density fluctuation or primordial curvature perturbation.

The observed E-type polarization power spectrum can be expressed as

$C_{l}^{E}(\nu)=\frac{2}{\pi} \int k^{2} d k\left\{\bar{x}_{\mathrm{H}}^{2} P_{\delta}(k)\left[{ }_{\delta} \Delta_{l}^{E}(k, \nu)\right]^{2}+P_{x}(k)\left[{ }_{x} \Delta_{l}^{E}(k, \nu)\right]^{2}\right\}$,

where $P_{\delta}(k)$ is the baryonic power spectrum and $P_{x}(k)$ is the ionization fraction power spectrum. Here we have defined $\bar{x}_{\mathrm{H}}$ to be the average ionization fraction. We ignore the cross-correlation between $\delta$ and $x_{\mathrm{H}}$. The baryon density fluctuation can be expressed as

$$
\delta\left(\boldsymbol{k}, \eta_{E}\right)=T(k) D\left(\eta_{E}\right) \zeta(\boldsymbol{k}),
$$

where $T(k)$ is the standard transfer function calculated by CMBFAST, ${ }^{9} D(\eta)$ is the linear theory growth function, and $\zeta(\boldsymbol{k})$

\footnotetext{
9 Available at http://www.cmbfast.org/.
} 
is the primordial curvature fluctuation produced by inflation. Thus, the baryonic power spectrum can be expressed as

$$
P_{\delta}\left(k, \eta_{E}\right)=T^{2}(k) D^{2}\left(\eta_{E}\right) P_{\zeta}(k),
$$

where $P_{\zeta}(k)$ is the scale-invariant primordial curvature power spectrum.

As previously mentioned, there will also be correlations in the ionization fraction fluctuations. The relative importance of the contributions from the baryonic power spectrum and the ionization fraction power spectrum depends on the mean ionization fraction, as well as on the topology of $\mathrm{H}$ II regions. The calculation of $P_{x}(k)$ is complicated by uncertainties about the ionizing source population and the radiative transfer of the ionizing radiation. There have been recent attempts to semianalytically model this power spectrum based on the halo model (McQuinn et al. 2005), which divides the power spectrum into contributions from the internal distribution of individual halos and the large-scale clustering of halos that are biased tracers of the linear baryonic matter power spectrum (Cooray \& Sheth 2002). The contribution from individual halos introduces Poisson fluctuations in the $\mathrm{H}$ II regions and dominates the power spectrum on small scales. On large scales, the halos are a biased tracer of the linear baryonic power spectrum. The transition between these two regimes occurs near the characteristic bubble size.

In this paper we focus on the contribution to the incident quadrupole from Poisson fluctuations in the number of $\mathrm{H}$ II regions. For simplicity and definitiveness we only include this effect at the end of reionization, namely, the surface of bubble overlap, where we can robustly calculate the size and number density of $\mathrm{H}$ II regions based on the general considerations of cosmic variance and light propagation delay ${ }^{10}$ (Wyithe \& Loeb $2004 \mathrm{~b}$ ). As long as the volume filling fraction of ionized bubbles is small, the $21 \mathrm{~cm}$ fluctuations are small, so most of the signal naturally originates around the time of bubble overlap anyway. We ignore the correlations of the ionization fraction due to large-scale biasing of the $\mathrm{H}$ II regions, as well as crosscorrelations between the baryon density and the ionization fraction. Biasing effects can only be important on scales above the characteristic size of the $\mathrm{H}$ II regions. However, at the end of reionization the characteristic size of the $\mathrm{H}$ II regions is extremely large ( $\sim 70$ comoving Mpc; Wyithe \& Loeb $2004 b)$. On these scales the amplitude of the baryonic power spectrum is quite small, so the biased $\mathrm{H}$ II region power spectrum will be modest at the end of reionization. Besides, these effects are primarily important on large angular scales, at which the conversion of the temperature brightness fluctuations to the necessary quadrupole anisotropies is suppressed; therefore, we find them to be below realistic instrument detection thresholds.

The observed fluctuations in the ionization fraction are caused by fluctuations in the local number of $\mathrm{H}$ II regions. Poisson statistics of pointlike objects is described by the spatial two-point correlation function,

$$
\left\langle\delta x\left(\boldsymbol{r}_{1}\right) \delta x\left(\boldsymbol{r}_{2}\right)\right\rangle=\frac{1}{\bar{n}\left(\boldsymbol{r}_{1}\right)} \delta^{(3)}\left(\boldsymbol{r}_{1}-\boldsymbol{r}_{2}\right),
$$

where $\bar{n}$ is the average number density of $\mathrm{H}$ II regions and $\delta x$ is the fluctuation in the ionization fraction. Since the $\mathrm{H}$ II regions

\footnotetext{
10 Note that inclusion of the light propagation delay is essential for the calculation of the size of an $\mathrm{H}$ ir bubble at the end of reionization, as viewed from the location of a scattering electron. Any calculation of the typical bubble size in a spacelike snapshot of the universe (as commonly done in the literature) is not adequate for this purpose.
}

have a finite size, we must convolve their density field with a window function

$$
\delta x\left(\boldsymbol{r}_{1}\right)=\int d^{3} \boldsymbol{r}_{2} W_{R}\left(\left|\boldsymbol{r}_{1}-\boldsymbol{r}_{2}\right|\right) \delta x\left(\boldsymbol{r}_{2}\right)
$$

where we assume a Gaussian window function for computational simplicity,

$$
W_{R}(r)=\frac{e^{-r^{2} / 2 R^{2}}}{\sqrt{(2 \pi)^{3}} R^{3}}
$$

The two-point correlation function is given by

$$
\begin{aligned}
& \left\langle\delta x\left(\boldsymbol{k}_{1}\right) \delta x^{*}\left(\boldsymbol{k}_{2}\right)\right\rangle=\int d^{3} \boldsymbol{r}_{1} d^{3} \boldsymbol{r}_{2} e^{i \boldsymbol{k}_{1} \cdot \boldsymbol{r}_{1}} e^{-i \boldsymbol{k}_{1} \cdot \boldsymbol{r}_{1}} \\
& \times \int d^{3} \boldsymbol{y}_{1} d^{3} \boldsymbol{y}_{2} \frac{e^{-\left|\boldsymbol{r}_{1}-\boldsymbol{y}_{1}\right|^{2} / 2 R^{2}}}{\sqrt{(2 \pi)^{3}} R^{3}} \frac{e^{-\left|\boldsymbol{r}_{2}-\boldsymbol{y}_{2}\right|^{2} / 2 R^{2}}}{\sqrt{(2 \pi)^{3}} R^{3}}\left\langle\delta x\left(\boldsymbol{y}_{1}\right) \delta x\left(\boldsymbol{y}_{2}\right)\right\rangle .
\end{aligned}
$$

Simplifying, we find

$$
\left\langle\delta x\left(\boldsymbol{k}_{1}\right) \delta x^{*}\left(\boldsymbol{k}_{2}\right)\right\rangle=\frac{1}{\bar{n}} \int d^{3} \boldsymbol{r}_{1} d^{3} \boldsymbol{r}_{2} e^{i \boldsymbol{k}_{1} \cdot \boldsymbol{r}_{1}} e^{-i \boldsymbol{k}_{1} \cdot \boldsymbol{r}_{1}} \frac{e^{-D^{2} / R^{2}}}{\sqrt{(2 \pi)^{3}} R^{3}},
$$

where we have defined $\boldsymbol{D}=\left(\boldsymbol{r}_{1}-\boldsymbol{r}_{2}\right) / 2$. As $R \rightarrow 0$, the Gaussian window function approaches $\delta^{(3)}\left(\boldsymbol{r}_{1}-\boldsymbol{r}_{2}\right)$, and then we find $P_{x}(k)=1 / \bar{n}$, where we define the ionization fraction power spectrum, $P_{x}(k)$, through $\left\langle\delta x\left(\boldsymbol{k}_{1}\right) \delta x^{*}\left(\boldsymbol{k}_{2}\right)\right\rangle=(2 \pi)^{3} \delta^{(3)}\left(\boldsymbol{k}_{1}-\boldsymbol{k}_{2}\right) P_{x}\left(k_{1}\right)$. We can now analytically calculate the two-point function for finite bubble size,

$$
P_{x}(k)=\frac{1}{\bar{n}} e^{-k^{2} R^{2}} .
$$

The translational invariance of $\left\langle\delta x\left(\boldsymbol{r}_{1}\right) \delta x\left(\boldsymbol{r}_{2}\right)\right\rangle$ guarantees that $\left\langle\delta x\left(\boldsymbol{k}_{1}\right) \delta x^{*}\left(\boldsymbol{k}_{2}\right)\right\rangle$ will be proportional to $\delta^{(3)}\left(\boldsymbol{k}_{1}-\boldsymbol{k}_{2}\right)$. The cutoff in the temperature brightness fluctuation power spectrum due to the finite bubble size will cause a corresponding decay in the polarization power spectrum for multipole index $l \gtrsim \eta_{R} / R$. Below this scale, the aforementioned signal due to baryon fluctuations will come to dominate. However, as we see in $\S 3$, this contribution to the signal is far below the instrumental noise detection threshold. Therefore, we are justified in ignoring it.

We assumed $\bar{n}(\boldsymbol{r})$ to be constant because of the large-scale homogeneity of the universe; the coordinate $\boldsymbol{r}$ is the comoving distance on the hypersurface of simultaneity corresponding to the time of reionization, not a coordinate labeling our past light cone. This Poisson fluctuation contribution to the polarization power spectrum corresponds to the intersection (within the region defined by the bubble window function) of the past light cones of the two electron scatterers, which both lie on our past light cone, on the hypersurface of simultaneity corresponding to the end of reionization. Figure 1 schematically shows this effect. On the right-hand side of the figure, scatterers along a given line of sight see $21 \mathrm{~cm}$ radiation from progressively larger shells because the elapsed time between emission and scattering for an electron increases for electrons closer to the observer. On the left-hand side of the figure, the shells of $21 \mathrm{~cm}$ radiation emission are displayed for two electrons along two lines of sight 


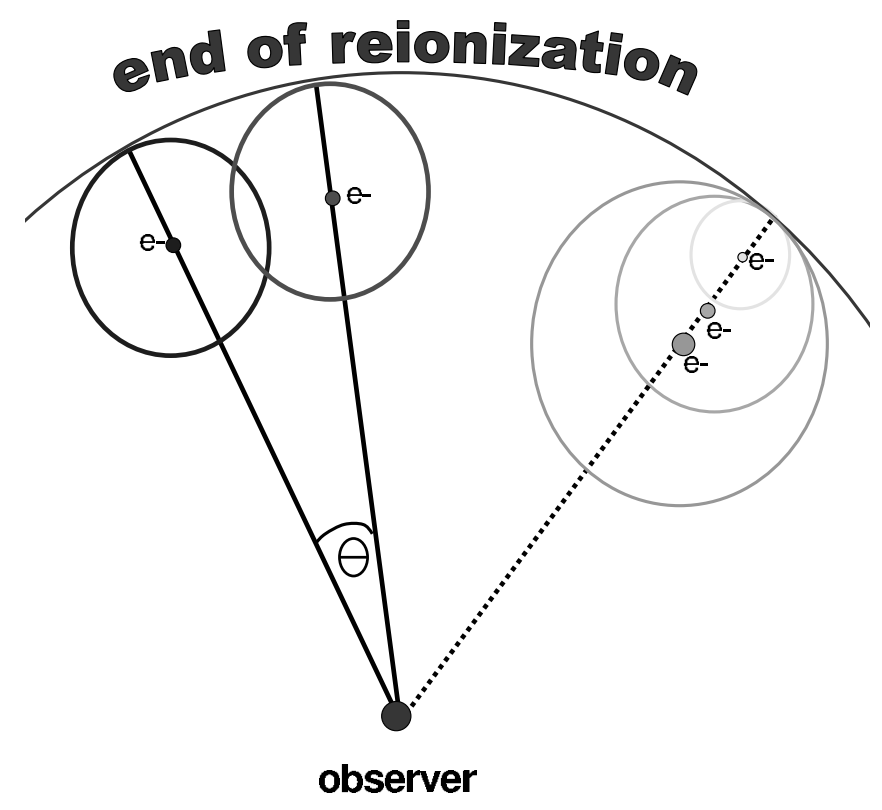

FIG. 1.-Schematic illustration of the scattering geometry. Each electron along the dotted (rightmost) line of sight scatters $21 \mathrm{~cm}$ radiation that was emitted just before the end of reionization at a redshift $z_{R}$ from a spherical shell around it. All emitting shells are tangent to the bounding shell around the observer. The $21 \mathrm{~cm}$ radiation emitted by the patchy $\mathrm{H}$ i distribution in these shells and scattered after reionization by the electrons at the shell centers will show polarization at a wavelength of $(21 \mathrm{~cm})\left(1+z_{R}\right)$. The two solid lines of sight on the left that are separated by an angle $\theta$ will show correlations in their polarization amplitudes due to scattering electrons with intersecting emission shells. [See the electronic edition of the Journal for a color version of this figure.]

separated by an angle $\theta$. These shells are distinct from the observer's shell because they correspond to past light cones of the electrons that intersect the hypersurface of simultaneity when reionization ended at different physical positions. In the figure, the shells for the two electrons intersect, and therefore the polarization produced along the two lines of sight will be correlated due to Poisson fluctuations.

At the end of reionization, which we define to be the surface of bubble overlap (Wyithe \& Loeb 2004b), the mean distance between $\mathrm{H}$ II regions is equivalent to the characteristic size of the bubbles. Thus, $\bar{n}$, the average bubble density, equals $1 / V$, where $V=4 \pi R^{3} / 3$ is the characteristic bubble volume. This is only true at the end of reionization; earlier on, during the process of reionization, the characteristic bubble size is smaller than the mean bubble separation. For our model, we find that the polarization power spectrum is given by

$$
C_{l}^{E}(\nu)=\frac{8 R^{3}}{3} \int k^{2} d k e^{-R^{2} k^{2}}\left[{ }_{x} \Delta_{l}^{E}(k, \nu)\right]^{2} .
$$

Since the brightness temperature fluctuations are the source of the quadrupole that produces polarization through Thomson scattering, the polarization and temperature signals should be correlated. Actually, only the $E$-type polarization is correlated with the temperature fluctuations, as parity prevents correlations with $B$-type polarization (Zaldarriaga \& Seljak 1997). Depending on the characteristics of reionization, this cross-correlation signal could be much easier to detect. For this reason the CMB temperature-polarization cross-correlation spectrum was first published by DASI (Degree Angular Scale Interferometer; Kovac et al. 2002) and WMAP (Kogut et al. 2003). It is straightforward to adapt the above formalism. The temperature transfer func- tion is again divided into contributions from the baryon density fluctuations,

$$
{ }_{\delta} \Delta_{l}^{T}(k, \nu)=j_{l}\left(k \eta_{E}\right)-j_{l}^{\prime \prime}\left(k \eta_{E}\right)
$$

and the ionization fraction fluctuations,

$$
{ }_{x} \Delta_{l}^{T}(k, \nu)=j_{l}\left(k \eta_{E}\right)
$$

Then the observed temperature polarization cross-correlation spectrum can be written as

$$
\begin{aligned}
C_{l}^{X}(\nu)=\frac{2}{\pi} \int\left\{k^{2} d k\right. & {\left[\bar{x}_{\mathrm{H}}^{2} P_{\delta}(k)_{\delta} \Delta_{l}^{E}(k, \nu)_{\delta} \Delta_{l}^{T}(k, \nu)\right.} \\
& \left.\left.+P_{x}(k)_{x} \Delta_{l}^{E}(k, \nu)_{x} \Delta_{l}^{T}(k, \nu)\right]\right\} .
\end{aligned}
$$

We have ignored effects due to the spectral response functions of the observing instruments. These are straightforward to incorporate into our formalism. Since we find that the dominant signal, which is produced by $\mathrm{H}$ II region Poisson fluctuations, is rather featureless and exponentially damped below scales of tens of arcminutes $\left(l>10^{3}\right)$, reasonable instrument bandwidths $(\Delta \nu \sim 0.4 \mathrm{MHz})$ will not substantially affect our results.

\section{RESULTS}

Based on the formalism developed in $\S 2$, we present numerical results for a variety of emission and reionization redshifts in order to understand how the signal depends on these parameters. We consider the simplest model in which reionization is assumed to be sudden, so the ionization fraction is a step function, and homogeneous, except for the effect of Poisson fluctuations of $\mathrm{H}$ II regions on the incident $21 \mathrm{~cm}$ quadrupole. This implies that the $21 \mathrm{~cm}$ power spectrum will simply be due to correlations in the baryonic density and that the gas is always completely neutral, $\bar{x}_{\mathrm{H}}=1$, when the redshift of the emission is greater than the redshift of reionization. When we observe emitted radiation from the redshift of reionization, we include the contribution from the Poisson fluctuations in the $\mathrm{H}$ II region distribution. Once the universe becomes reionized, it does so uniformly. Our approximate treatment ignores scattering due to the patchiness of the universe while it is only partially ionized. We adopt for our first treatment this simplified model because it has the smallest number of free parameters; more complicated models with uncertain astrophysical parameters can be considered in the future.

Figure 2 compares the temperature brightness power spectrum $\left(C_{l}^{T}\right)$ and the polarization power spectrum $\left(C_{l}^{E}\right)$ due to baryon density fluctuations for $z_{E}=20$ and $z_{R}=17$. The features in $C_{l}^{T}$ and $C_{l}^{E}$ are quite different. In addition to being at a lower amplitude, $C_{l}^{E}$ peaks and begins to oscillate and decay at high $l$. The polarization transfer function, defined in equation (1), is the lineof-sight integral of two spherical Bessel functions, weighted by the visibility function and additional factors due to the polarization spin lowering operators. The properties of the spherical Bessel function are well known: when $x \ll 1, j_{l}(x) \sim x^{l}$, it has a peak at $x \sim l$, and then at large $x$ it oscillates and decays as $j_{l}(x) \sim \sin (x-\pi l / 2) / x$. We use these features to understand the behavior of $C_{l}^{E}$.

Figure 3 shows the polarization power spectrum for various reionization redshifts, $z_{R}=17,15,10$, and 6 (top to bottom), and a single emission redshift, $z_{E}=20$. The basic physical processes become clear by comparing these various power spectra. We first consider the angular position of the peak in the polarization 


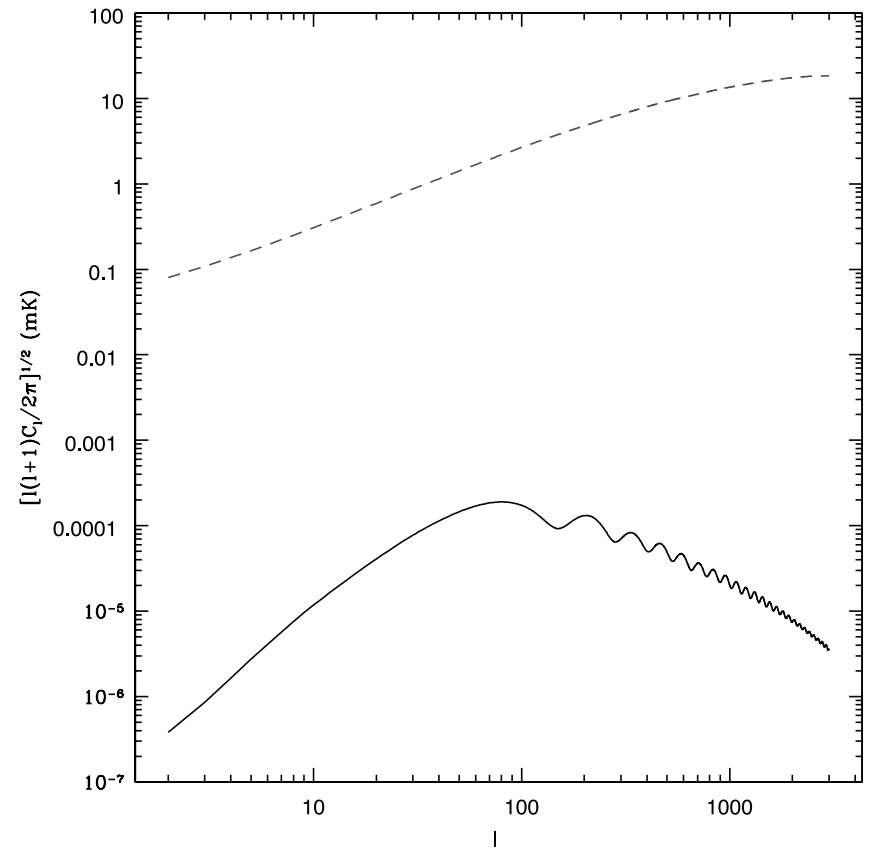

FIG. 2.-Brightness temperature (dashed line) and polarization (solid line) due to baryon density fluctuations for emission redshift $z_{E}=20$ and reionization redshift $z_{R}=17$. [See the electronic edition of the Journal for a color version of this figure.]

power spectra. The spherical Bessel function $j_{l}(k \eta)$ peaks at $l \sim k \eta$ and likewise $j_{2}\left[k\left(\eta_{E}-\eta\right)\right]$ at $2 \sim k\left(\eta_{E}-\eta\right)$. These two constraints imply that the polarization power spectrum peaks at

$$
l_{\text {peak }} \sim \frac{2 \eta_{R}}{\eta_{E}-\eta_{R}},
$$

where $\eta_{E}$ and $\eta_{R}$ are the comoving distances to the redshifts of emission and reionization, respectively. Here we have effectively assumed an infinitely sharp visibility function. The inclusion of the altered free-streaming term, due to peculiar velocities, only has a minor effect on this argument. Thus, changing $z_{R}$, for a given $z_{E}$, will shift the power spectrum features to higher $l$. This is clearly observed in Figure 3, and numerically the peak locations agree with equation (26).

The oscillatory features are due to the free-streaming of the monopole brightness temperature fluctuations. The incident quadrupole on a scatterer, equation (9), contains the spherical Bessel function, $j_{2}\left[k\left(\eta_{E}-\eta\right)\right]$, which describes how a monopole fluctuation of wavevector $k$ at $\eta_{E}$ becomes a quadrupole fluctuation at $\eta$. The projection of this oscillatory function, the free-streaming quadrupole, on the sky causes the features in Figure 3. These are not the analogs of the acoustic peaks observed in the primary temperature anisotropies of the CMB (Barkana \& Loeb 2005c). Both are caused by projecting an oscillatory function on the sky; however, the acoustic oscillations originate from pressure waves in the baryon-photon fluid prior to recombination.

The decay of $C_{l}^{E}$ at high $l$ is explained by oscillatory cancellation of the line-of-sight integration. The two spherical Bessel functions would have had different phases and therefore integrated to zero if the range of integration was infinite. When the wavelength of the perturbation becomes comparable in size to the width of the visibility function, this effect begins to become important. Since the visibility function becomes progressively more peaked at higher reionization redshifts, progressively smaller intervals along the line of sight contribute to the trans-

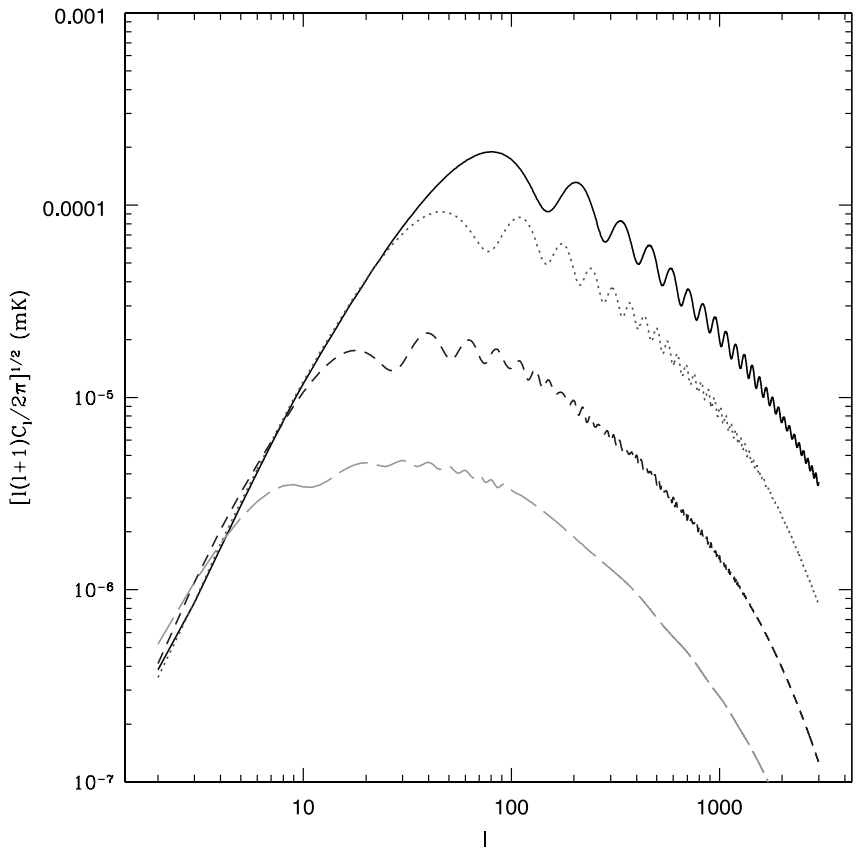

FIG. 3.-Polarization power spectrum sourced by baryon density fluctuations for an emission redshift $z_{E}=20$ and reionization redshift $z_{R}=17$ (solid line), $z_{R}=15$ (dotted line), $z_{R}=10$ (dashed line), and $z_{R}=6$ (long-dashed line). [See the electronic edition of the Journal for a color version of this figure.]

fer function, and therefore there is less damping. We observe this phenomenon in Figure 3, as the high-l tail decays less and retains oscillatory features for the curves corresponding to the higher reionization redshifts. If the change in the ionization fraction is not sudden as we have assumed but rather gradual, then these oscillatory features would be smoothed out.

In these examples we have assumed that reionization is instantaneous, while in reality reionization must be gradual (Furlanetto \& Loeb 2005). As mentioned in $\S 2.2$, the adopted reionization history will imprint a signature on the polarization power spectrum. Next we demonstrate that the polarization power spectrum sourced by baryon density fluctuations and produced for a given gradual reionization history can be well approximated (within the appropriate cosmic variance and instrumental noise error bars) by an instantaneous reionization history (with the total optical depth not being necessarily the same in the two reionization histories). In the bottom panel of Figure 4 the polarization power spectra sourced by baryon density fluctuations are displayed for the instantaneous and gradual reionization histories plotted in the top panel. While there are differences between the two reionization histories, they are fairly modest compared to the much greater instrumental noise and model uncertainties. The use of the simple model of instantaneous reionization is therefore well justified for the scope of this paper.

Figure 5 displays the polarization power spectra sourced by Poisson fluctuations of an $\mathrm{H}$ II region, for different redshifts of emission and reionization: $z_{E}=z_{R}=30$ (solid line), $z_{E}=z_{R}=25$ (dotted line), $z_{E}=z_{R}=17$ (dashed line), $z_{E}=z_{R}=10$ (longdashed line), and $z_{E}=z_{R}=6$ (dot-dashed line). The theoretical detection threshold of SKA is also shown for a 1 month integration time and a $1 \mathrm{yr}$ integration time. We can understand the basic features of the polarization power spectra by inspecting equation (22). The amplitude of the polarization power spectra is proportional to the characteristic volume of an $\mathrm{H}$ in bubble at the time of bubble overlap. If reionization completes at a higher redshift, then the $\mathrm{H}$ II bubbles are smaller and therefore 

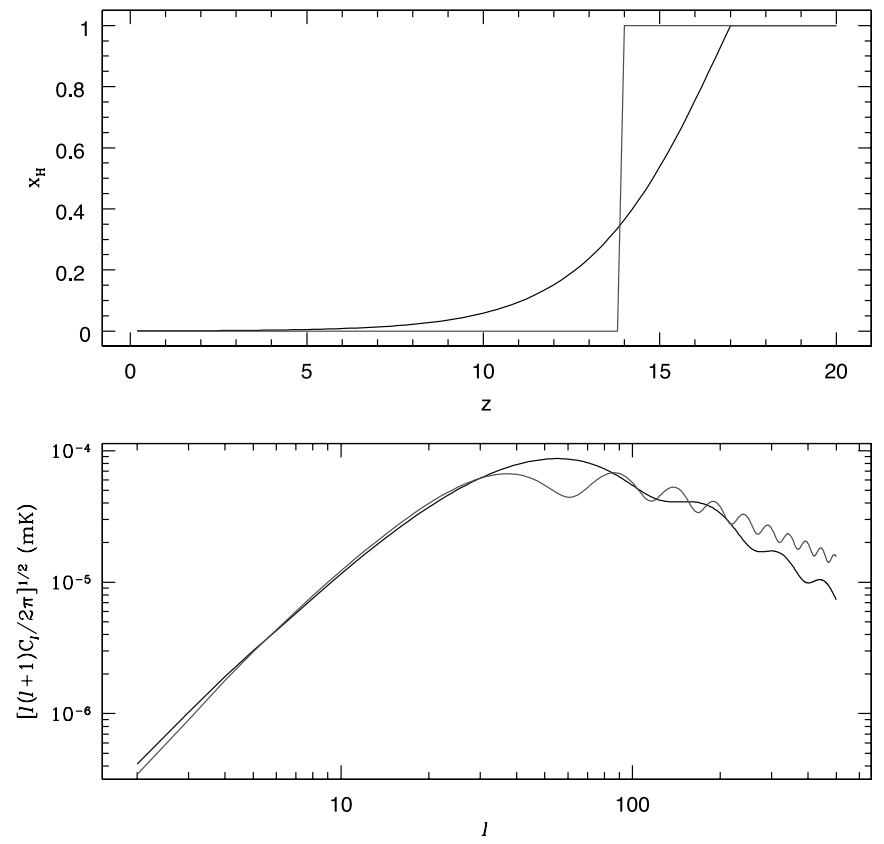

FIG. 4.-Top: Gradual (black line) and instantaneous (gray line) reionization histories appropriate for the polarization power spectra displayed in the bottom panel. Bottom: Polarization power spectra displayed for the instantaneous (gray line) and gradual (black line) reionization histories assuming that the $21 \mathrm{~cm}$ fluctuations are sourced by density inhomogeneities. [See the electronic edition of the Journal for a color version of this figure.]

more numerous at the surface of bubble overlap, which defines the end of reionization (Wyithe \& Loeb 2004b). Subsequently, this reduces the level of the Poisson fluctuations, which is inversely proportional to the $\mathrm{H}$ II region number density. The window function related to the finite size of a typical $\mathrm{H}$ II region produces an exponential decay in the power spectrum on smaller spatial scales. Thus, the power spectra corresponding to higher reionization redshifts, and therefore smaller characteristic sizes, begin to exponentially decay at higher values of $l$.

In Figure 5 the amplitude of the polarization power spectra increases with increasing reionization redshift. This conflicts with the naive expectation that the polarization power spectra will be smaller at high redshift, since the Poisson fluctuations are smaller. The reason is that the decrease in the characteristic $\mathrm{H}$ II region size is rather gradual, and the visibility function is significantly increasing at high redshifts. This is the main reason for the fact that the polarization power spectra with low reionization redshifts have lower amplitudes. At yet higher redshifts a turnover does occur, and the decrease in characteristic $\mathrm{H}$ II region size overcomes the increase in optical depth.

The polarization power spectrum detection threshold due to instrument noise is included on Figure 5 for the Square Kilometre Array (SKA), which is the next-generation low-frequency radio interferometer. We adopt the power spectrum detection threshold model of Zaldarriaga et al. (2004) with updated parameters. We assume an instrument bandwidth of $\Delta \nu \sim 0.4 \mathrm{MHz}$ throughout the paper; increasing the bandwidth will decrease the instrument detection threshold as $\Delta \nu^{-1 / 2}$. In reality, the relevant power spectrum error bars for a radio interferometer are a complicated function of both the exact instrumental design and the analysis method, so the detection thresholds in this work should be viewed as a crude estimate (assuming that the foregrounds can be properly removed). Measurements of the proximity effect around highredshift quasars imply that the end of reionization, i.e., the surface of bubble overlap, is near $z=6$ (Wyithe \& Loeb 2004a).

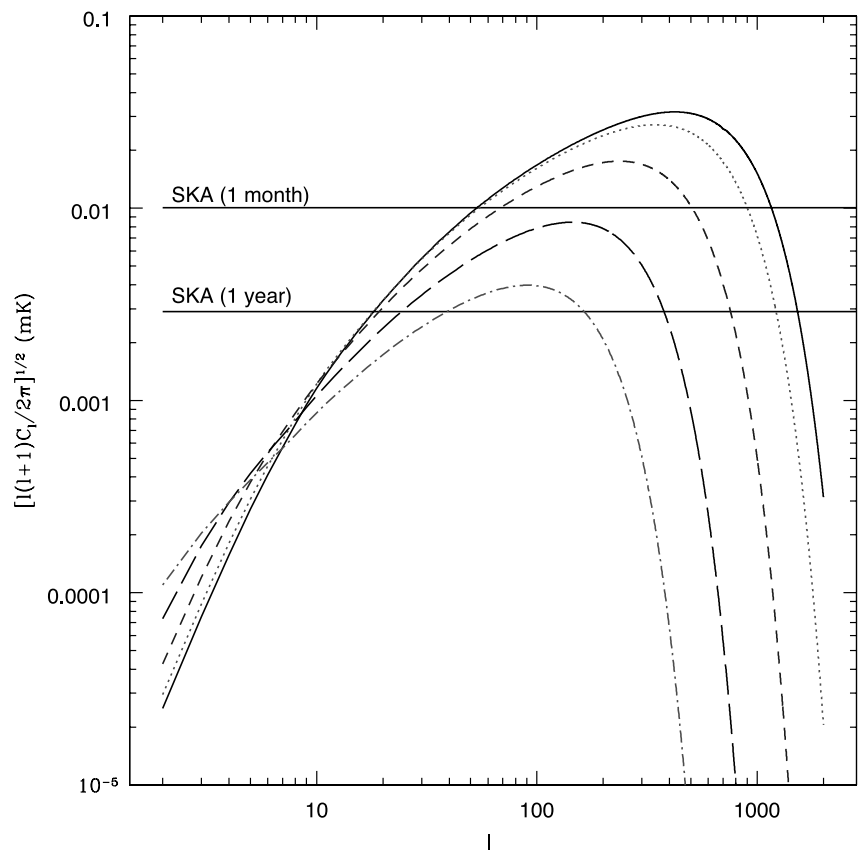

FIG. 5.-Polarization power spectrum sourced by Poisson fluctuations of $\mathrm{H}$ II regions for an emission and reionization redshift $z_{E}=z_{R}=30$ (solid line), $z_{E}=z_{R}=25$ (dotted line), $z_{E}=z_{R}=17$ (dashed line), $z_{E}=z_{R}=10$ (longdashed line), and $z_{E}=z_{R}=6$ (dot-dashed line). The theoretical detection threshold of SKA is also shown for a 1 month integration time and a $1 \mathrm{yr}$ integration time with a bandwidth of $0.4 \mathrm{MHz}$. [See the electronic edition of the Journal for a color version of this figure.]

We find that the polarization signal from even a redshift as low as $z_{E}=z_{R}=6$ is detectable with a $1 \mathrm{yr}$ integration time. A higher redshift of reionization will only increase the amplitude of the polarization power spectrum.

In $\S 2$ we predicted that the temperature polarization crosscorrelation spectrum should be significantly larger than the polarization power spectrum. Since the polarization power spectrum can only be detected by a year-long integration with a nextgeneration instrument, we compute the cross-correlation spectrum and compare its amplitude to the noise detection thresholds of upcoming instruments. Figure 6 displays the temperaturepolarization cross-correlation spectra, sourced by $\mathrm{H}$ in region Poisson fluctuations, for different redshifts of emission and reionization: $z_{E}=z_{R}=30$ (solid line), $z_{E}=z_{R}=25$ (long-dashed line), $z_{E}=z_{R}=17$ (dashed line), $z_{E}=z_{R}=10$ (dotted line), and $z_{E}=z_{R}=6$ (dot-dashed line). Also shown are the instrument detection thresholds for SKA for 1 month of integration time, LOFAR for $1 \mathrm{yr}$, and MWA for $1 \mathrm{yr}$. We can understand how the features of these spectra differ from the polarization power spectra of Figure 5 by comparing the formula for the temperature polarization cross-correlation spectrum (eq. [25]) with the formula for the polarization power spectrum (eq. [22]).

There are two obvious differences between the spectra. The temperature polarization cross-correlation spectra have larger amplitudes and shallower slopes on large scales. Clearly, the polarization transfer function (eq. [1]) has a smaller amplitude than the temperature transfer function (eq. [23]) because of the low optical depth, as well as the effects of the quadrupole freestreaming and conversion to $E$-type polarization. The steep largescale slope observed in the polarization power spectra (Figs. 3-5) results from the quadrupole nature of the production of polarization through Thomson scattering (Hu \& White 1997). Each polarization transfer function roughly scales as $l^{2}$ on large scales, so the polarization power spectrum should naturally be steeper 


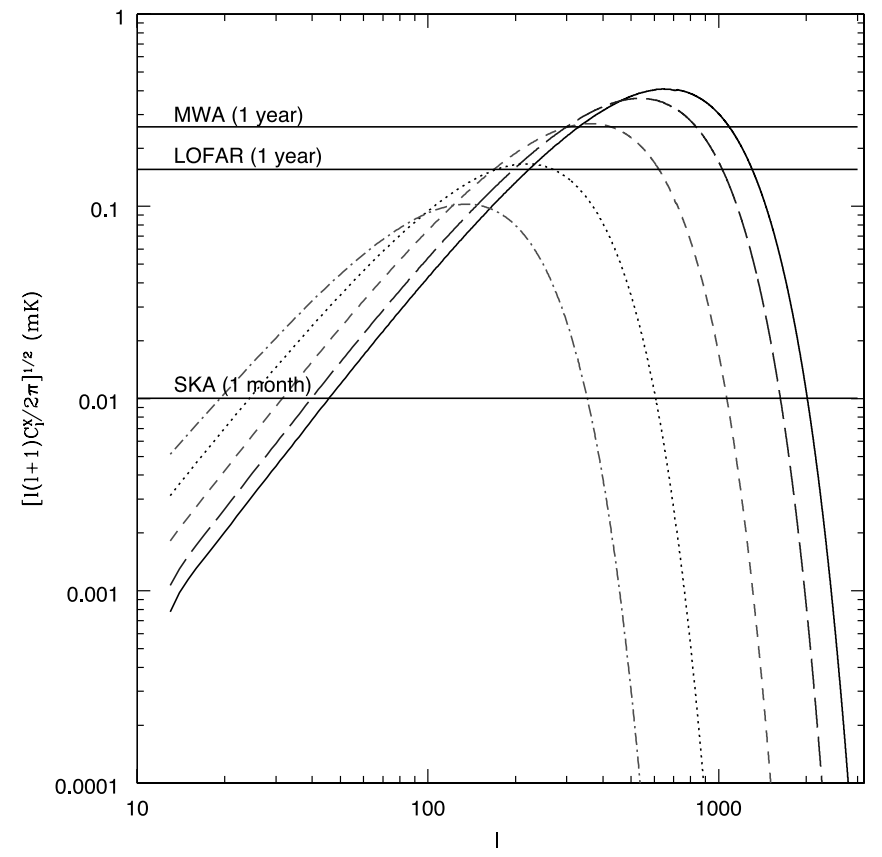

FIG. 6.-Temperature-polarization cross-correlation spectra sourced by Poisson fluctuations of $\mathrm{H}$ II regions for an emission and reionization redshift $z_{E}=z_{R}=30$ (solid line), $z_{E}=z_{R}=25$ (long-dashed line), $z_{E}=z_{R}=17$ (dashed line), $z_{E}=z_{R}=10$ (dotted line), and $z_{E}=z_{R}=6$ (dot-dashed line). The theoretical detection threshold of SKA is shown for 1 month, LOFAR for $1 \mathrm{yr}$, and MWA for $1 \mathrm{yr}$ of integration time, all with a bandwidth of $0.4 \mathrm{MHz}$. [See the electronic edition of the Journal for a color version of this figure.]

than the temperature polarization cross-correlation on these scales. Once again, on small scales we observe the same exponential decay caused by the $\mathrm{H}$ II region window function. We find that even if reionization ended at $z_{R}=6$, this signal should be easily detectable by SKA and very close to the detection threshold for LOFAR and MWA. If new observations of high-redshift quasars imply that the surface of bubble overlap occurred at a higher redshift, then the detectability of the temperature polarization crosscorrelation signal increases for LOFAR and MWA. The result is very encouraging because it is based on simple analytical considerations that are robust to variations in astrophysical parameters. These uncertain parameters are all lumped into the value of $z_{R}$ in our formulation.

\section{DISCUSSION}

Figure 6 implies that the future Square Kilometre Array (SKA) will have sufficient instrument sensitivity to detect the temperature polarization cross-correlation power spectrum of redshifted $21 \mathrm{~cm}$ fluctuations. The forthcoming Mileura Widefield Array (MWA) and the Low Frequency Array (LOFAR) could also have the sensitivity to detect the predicted signal. If new observations of high-redshift quasars push this redshift of bubble overlap to higher values, then the possibility of a detection increases. The practical feasibility of such a detection will be better known within a few years, as soon as the first polarized foreground maps are produced by LOFAR or MWA. The instrumental detection thresholds used here are based on simplifications and should only be interpreted as rough estimates. However, the results shown in Figure 6 portray the optimistic forecast that the polarization signal is sufficiently large for it to be detectable.

We identified the dominant producer of polarization to be Thomson scattering of an incident quadrupole moment of the $21 \mathrm{~cm}$ radiation. The $21 \mathrm{~cm}$ quadrupole is sourced by the freestreaming of brightness fluctuations from either correlated baryonic density fluctuations or Poisson fluctuations of $\mathrm{H}$ II regions. The amplitude of these Poisson fluctuations can be robustly predicted based on the general considerations of cosmic variance and light propagation delay at the end of reionization. We have found that for a reionization redshift of $z_{R}=6$, which is suggested by the observations of the Gunn-Peterson effect, the temperature polarization cross-correlation spectrum amplitude due to Poisson fluctuations should be of the order of $\sim 0.1 \mathrm{mK}$ and the polarization power spectrum amplitude $\sim 3 \mu \mathrm{K}$. Strong cross-correlation between the brightness temperature and polarization of the $21 \mathrm{~cm}$ radiation is generic for Thomson scattering. The cross-correlation will be weaker, for example, if the polarization fluctuations are sourced by magnetic fields whose distribution is spatially uncorrelated with the $21 \mathrm{~cm}$ brightness fluctuations.

Since Thomson scattering in a nearly uniform medium produces $E$-type polarization and is achromatic, its unique fingerprints can be separated from foregrounds associated with polarized synchrotron emission. Faraday rotation of the signal as it propagates through the IGM will modify the above conclusion; however, the frequency dependence of the effect is precisely known, and techniques developed to eliminate foregrounds could be adapted to reconstruct the original $E$-type polarization signal. It is highly unlikely that any foreground source would produce a polarized signal that only contains $E$-type polarization and that correlates with the cosmic brightness fluctuations. This could be used as a stringent test against foreground contamination and instrument systematics. A similar test has been adopted to check whether the reconstructed signal from weak gravitational lensing surveys is contaminated by instrumental systematics and has proven to be extremely useful (Refregier 2003). For upcoming experiments that might not have the sensitivity to observe the polarized $21 \mathrm{~cm}$ signal from the high-redshift IGM, the inferred signal should not correlate with any observed polarization. This can be used to further eliminate the large foregrounds that could contaminate the high-redshift signal that is sought after.

We would like to thank Matias Zaldarriaga, Oliver Zahn, Matt McQuinn, Chris Hirata, Miguel Morales, Steve Furlanetto, and Asantha Cooray for useful conversations. This work was supported in part by NASA grants NAG5-13292 and NNG 05GH54G and NSF grants AST 00-71019 and AST 02-04514 (for A. L.).
Barkana, R., \& Loeb, A. 2001, Phys. Rep., 349, 125

2002, ApJ, 578, 1

2004, ApJ, 609, 474

2005a, ApJ, 624, L65

2005b, ApJ, 626, 1

. 2005c, MNRAS, 363, L36
Bharadwaj, S., \& Ali, S. S. 2004, MNRAS, 352, 142

\section{REFERENCES}

Carilli, C. L., Gnedin, N. Y., \& Owen, F. 2002, ApJ, 577, 22

Cooray, A., \& Furlanetto, S. 2005, MNRAS, 359, L47

Cooray, A., \& Sheth, R. 2002, Phys. Rep., 372, 1

Di Matteo, T., Perna, R., Abel, T., \& Rees, M. 2002, ApJ, 564, 576

Field, G. B. 1959, ApJ, 129, 525

Furlanetto, S. R., \& Loeb, A. 2001, ApJ, 556, 619

. 2002, ApJ, 579, 1 
Furlanetto, S. R., \& Loeb, A. 2005, ApJ, 634, 1

Furlanetto, S. R., Zaldarriaga, M., \& Hernquist, L. 2004, ApJ, 613, 16

Gnedin, N. Y. 2004, ApJ, 610, 9

Haiman, Z., \& Holder, G. P. 2003, ApJ, 595, 1

Hogan, C. J., \& Rees, M. J. 1979, MNRAS, 188, 791

Holder, G. P., Haiman, Z., Kaplinghat, M., \& Knox, L. 2003, ApJ, 595, 1

Hu, W. 2000, ApJ, 529, 12

Hu, W., \& White, M. 1997, Phys. Rev. D, 56, 596

Kogut, A., et al. 2003, ApJS, 148, 161

Kosowsky, A., \& Loeb, A. 1996, ApJ, 469, 1

Kosowsky, A., Kahniashvili, T., Lavrelashvili, G., \& Ratra, B. 2005, Phys. Rev. D, 71, 043006

Kovac, J. M., Leitch, E. M., Pryke, C., Carlstrom, J. E., Halverson, N. W., \& Holzapfel, W. L. 2002, Nature, 420, 772

Kulsrud, R. M., Cen, R., Ostriker, J. P., \& Ryu, D. 1997, ApJ, 480, 481

Loeb, A., \& Zaldarriaga, M. 2004, Phys. Rev. Lett., 92, 211301

Madau, P., Meiksin, A., \& Rees, M. J. 1997, ApJ, 475, 429

McQuinn, M., Furlanetto, S. R., Hernquist, L., Zahn, O., \& Zaldarriaga, M. 2005, ApJ, 630, 643

Mesinger, A., \& Haiman, Z. 2004, ApJ, 611, L69

Morales, M. F., \& Hewitt, J. 2004, ApJ, 615, 7

Oh, S. P., \& Mack, K. J. 2003, MNRAS, 346, 871
Refregier, A. 2003, ARA\&A, 41, 645

Scott, D., \& Rees, M. J. 1990, MNRAS, 247, 510

Seljak, U., \& Zaldarriaga, M. 1996, ApJ, 469, 437

Shapiro, P. R., Iliev, I. T., \& Raga, A. C. 2004, MNRAS, 348, 753

Sokasian, A., Yoshida, N., Abel, T., Hernquist, L., \& Springel, V. 2004, MNRAS, 350, 47

Spergel, D. N., et al. 2003, ApJS, 148, 175

Sunyaev, R. A., \& Zel'dovich, I. B. 1975, MNRAS, 171, 375

Tozzi, P., Madau, P., Meiksin, A., \& Rees, M. J. 2000, ApJ, 528, 597

Vallée, J. P. 2004, NewA Rev., 48, 763

Wouthuysen, S. A. 1952, AJ, 57, 31

Wyithe, J. S. B., \& Loeb, A. 2003, ApJ, 586, 693 2004a, Nature, 427, 815 2004b, Nature, 432, 194 2004c, ApJ, 610, 117 2005, ApJ, 625, 1

Zahn, O., Zaldarriaga, M., Hernquist, L., \& McQuinn, M. 2005, ApJ, 630, 657 Zaldarriaga, M. 1997, Phys. Rev. D, 55, 1822

Zaldarriaga, M., Furlanetto, S. R., \& Hernquist, L. 2004, ApJ, 608, 622

Zaldarriaga, M., \& Seljak, U. 1997, Phys. Rev. D, 55, 1830 1998, Phys. Rev. D, 58, 023003

Zygelman, B. 2005, ApJ, 622, 1356 